\title{
Stereotactic limbic leucotomy: surgical technique
}

\author{
Alan Richardson \\ F.R.C.S.
}

\begin{abstract}
Department of Neurosurgery, St George's Hospital at Atkinson Morley's Hospital, Wimbledon, SW20 0NE
\end{abstract}

\section{Summary}

The requirements for modern psychosurgery are safety and accuracy.

Stereotactic techniques give the geometric accuracy and stimulation gives physiological information, which is important in determining lesion sites or at least lesion symmetry.

The process whereby focal brain destruction is produced is ideally by a freezing probe, but equally effectively by coagulation. A number of small lesions is thus required. This at present is unavoidable if side effects are to be obviated. Careful continuing assessment of results is necessary to validate any surgical procedure.

THE basic neuro-anatomical and neuro-physiological concepts in relation to leucotomy for the treatment of mental illness have advanced over the last 20 years. This has been correllated with early surgical experience and has led to the modern approach to psychosurgery. The frontal lobes were the traditional sites for surgical interference in the majority of procedures, but a clear understanding of the relationships of the medial and orbital surfaces of the frontal lobe to the limbic system has not only modified views regarding the appropriate sites for lesion making but also helped to define more accurately the extent of the necessary procedures.

As Livingston (1969) has pointed out 'we can visualize a great fronto-limbic-hypothalamic-midbrain behavioural axis in which two somewhat parallel circuits of activity stand out. The medial frontalcingulate-hippocampal and the other the orbital frontal-temporal-amygdalar, both playing down on the hypothalamus and brain stem. The medial frontal-cingulate-hippocampal circuit is closed via fornix-mamillary-anteriorthalamic-frontal connections, while the orbital frontal-temporal-amygdalar circuit is closed via amygdalar-dorsomedialthalamic-frontal connections.' It must not be assumed, however, that these circuits are discrete, for Nauta (1962) has made the point that their efferent conducting systems are quite comparable, suggesting that they may form part of a massive linked system.

The interrelationships of the frontal lobe to the limbic system was taken further by Nauta (1971) when he suggested that the frontal lobe both modulated and monitored limbic activity. Lewin (1961) viewed the medial limbic circuit as a potential reverberating system to explain the beneficial effect of cingulectomy on obsessional states. Finally, one might propose that the medial and perhaps orbital surfaces of the frontal lobe may be played upon by any disturbance in a reverberating limbic circuit and in the words of Papez (1937) 'add emotional colouring to the psychic process'.

These rather simple, and perhaps oversimplified, considerations form the basis of our current surgical approach to the more severe and intractable obsessional disorders which have failed to respond to adequate psychiatric treatment. The objective is to interrupt the cingulum bundle at the probable points of circuit closure and to produce small destructve lesions in the medial portion of the posterior orbital white matter of the frontal lobe at the point of concentration of fronto-limbic interconnections.

\section{Technique}

Absolute requirements for modern psychosurgery are safety, so that operative mortality is virtually zero and operative morbidity is negligible. Lesion placement must be accurate and on sound anatomical and physiological bases so that the lesion size can be reduced to a minimum and so that valid comparison can be made on clinical analysis to assess the efficacy of given lesion patterns. Finally, the method of lesion production should be free from complication or secondary effects, the lesion should be complete at the time of operation and the lesion should not subsequently alter in size or character. These criteria virtually exclude any freehand method of surgery and dictate the necessity for an accurate stereotactic technique with the ability to check the site of lesion making and a predictable method for circumscribed lesion production.

\section{Procedure}

The operation is usually performed under general anaesthesia. In order to ensure absolute sterility a 
full head shave is required. The reasons for this are carefully explained to the patient and the hair removal performed after the patient is anaesthetized. We use the stereotactic frame designed by Professor Leksell which is illustrated in Fig. 1. It is basically a geometrical open box with radio-opaque graduations on each of the side arms. Upon this frame is shown the semi-circular arm which carries the electrode or probe guidance system and is so designed that after appropriate calculation the tip of the electrode or probe can be placed at any spatial point within the frame to an accuracy of $1 \mathrm{~mm}$.

At the commencement of the operation the frame, minus the semi-circular arm, is fixed to the patient's head by drill points which just perforate the outer table of the skull, ensuring rigid fixation to the skull and thus literally fixing the geometric frame in relation to the skull and its contents. With the patient in the supported sitting position a lumbar air encephalogram is performed and X-rays taken in the lateral and antero-posterior projections. The X-ray tube carries a precision optical bench which attaches to the frame, thus ensuring an unvarying relationship between the $X$-ray beam, the frame and the patient's head. The $\mathrm{X}$-ray photographs show the graduations on the frame, the bony structures of the skull and the cerebral ventricles and main subarachnoid pathways outlined by the injected air.

\section{Selection of target areas}

The standard X-ray projections are shown in Figs. 2 and 3. Both frontal horns and bodies of the lateral ventricles are demonstrated as is the midline third ventricle. Potential target areas in the frontal lobe are selected in relation to bony and ventricular landmarks, a line being drawn vertically at a point $1.5 \mathrm{~cm}$ anterior to the base of the anterior clinoid processes and a second vertical drawn at a point $1 \mathrm{~cm}$ posterior to the tip of the frontal horn. The initial target areas are usually plotted along the anterior of these two lines, defining three areas in the form of a triangle with its apex superiorly. The medial areas are 1 and $1.5 \mathrm{~cm}$ above the anterior fossa floor and $6 \mathrm{~mm}$ from the midline of the third ventricle, whereas the lateral frontal area is $1 \mathrm{~cm}$ above the anterior fossa floor and $14 \mathrm{~mm}$ from the midline.

For the cingulate lesions two pairs of areas are defined, being anterior and posterior. The posterior lesion area is plotted on a vertical line $3 \mathrm{~cm}$ posterior to the tip of the frontal horn of the ventricle and $5 \mathrm{~mm}$ above the body of the ventricle. The lateral offset is at the border of the ventricle and the medial area is $8 \mathrm{~mm}$ medial to this. The anterior lesion areas are $12 \mathrm{~mm}$ in front of the posterior cingulate sites.

These various zones are plotted on the radiographs and the co-ordinates for each of them calculated in three dimensions from the stereotactic frame

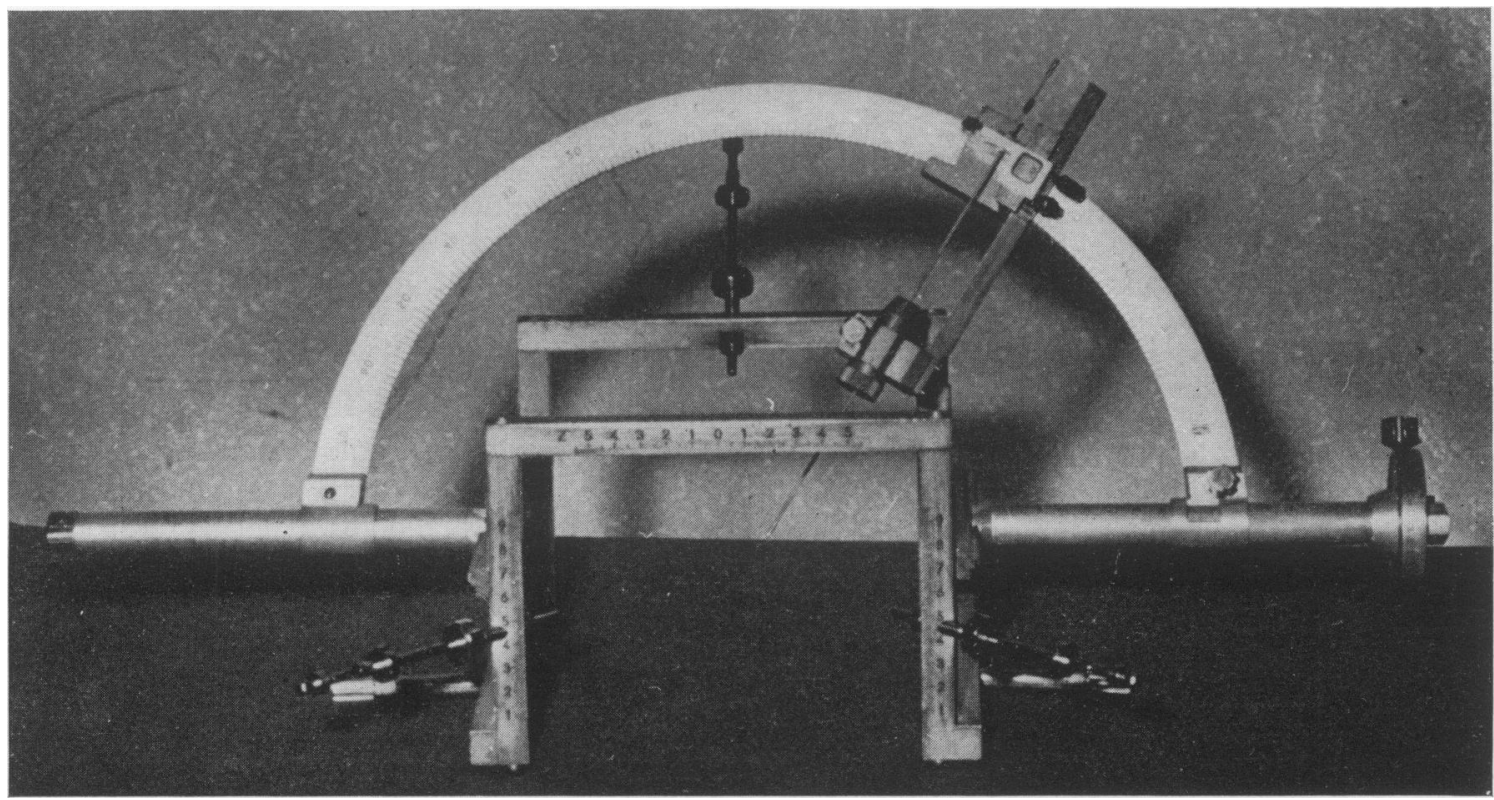

FIG. 1. Illustrating stereotactic frame with arc and electrode carrier attached and showing a stimulating electrode in position. 


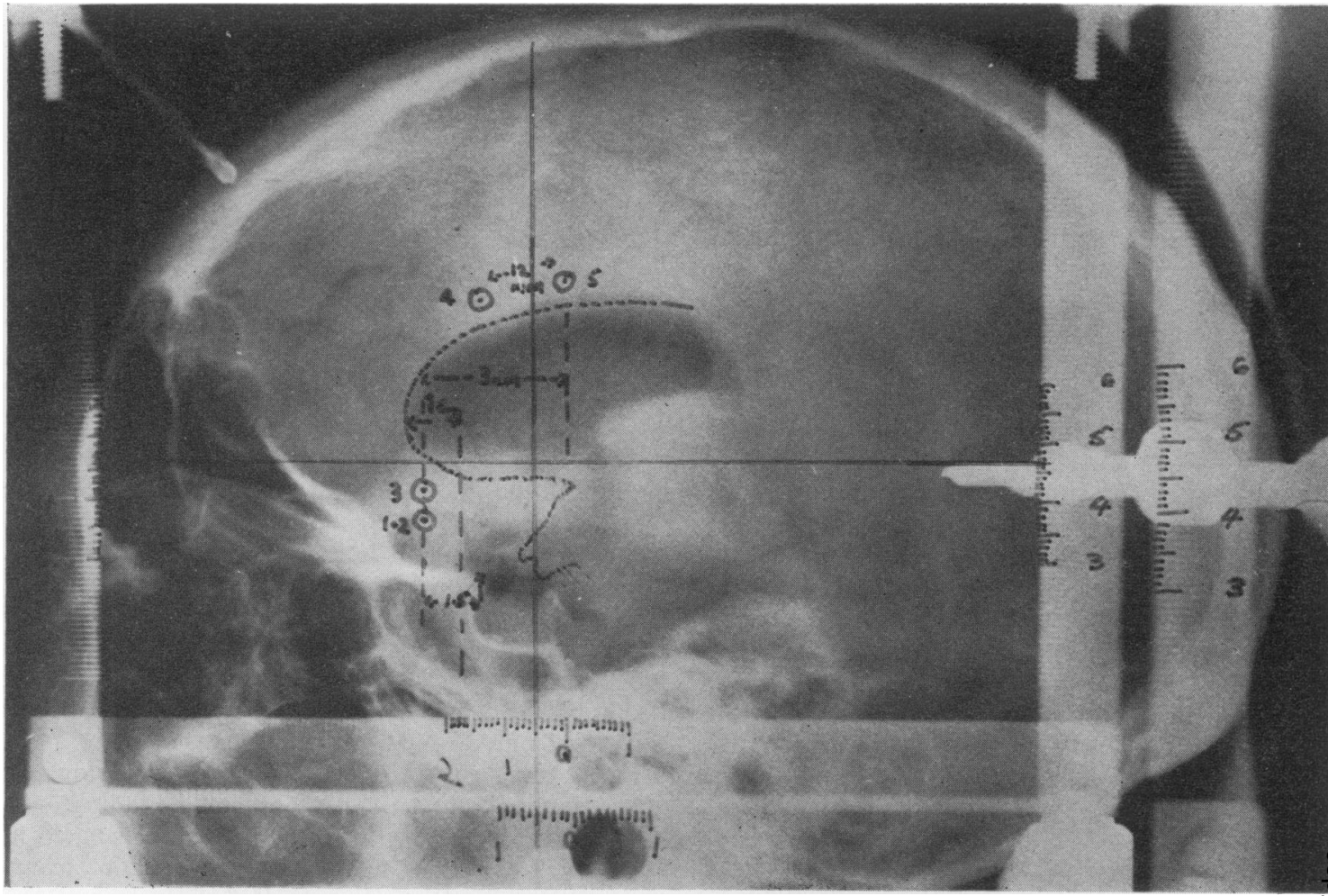

FIG. 2. Lateral radiograph showing stereotactic frame attached to the skull with the ventricular system outline by air. The target zones 1,2 and 3 in the frontal lobe are shown, as are target zones 4 and 5 for the cingulum bundle.

graduations after making due allowance for magnification or rotational factors.

\section{Localization of targets}

At this stage one has a series of seven accurately defined target zones in each hemisphere, and by placing the arc on the frame at the appropriate coordinates it is possible to pass an electrode or probe through a skull burrhole to the pre-selected target point. Advantage is then taken of the known electrical responses of parts of the limbic system and its projections to define the elective target sites. A bipolar stimulating electrode is inserted to the first target area and stimulation performed with alternating current at $60 \mathrm{~Hz}$ at $8-10 \mathrm{~V}$ for $15 \mathrm{sec}$. During this part of the procedure continuous recording of respiration, heart rate, skin resistance and forearm blood flow is in progress. The usual response in an active area is apnoea with less constant changes in pulse amplitude and forearm blood flow. The area of responsiveness is usually circumscribed, and moving as little as $4 \mathrm{~mm}$ from it may abolish the response. If no or little response results the target area is changed by a fixed distance and such studies continued until an area of suitable activity is identified. An arbitrary limit of three locations is made to avoid the trauma of repeated electrode insertion. It is rare to fail to find an active area within these limits, and it is similarly not common to find the target sites in the two hemispheres to be asymmetrical.

Careful monitoring of the general anaesthesia has abated any interference with the effects of stimulation, which are in any case only performed when the readings show the physiological state to be steady.

\section{Methods of focal white matter interruption}

Interruptive lesions of white fibre bundles can be made by a knife, wire loop, a blunt cannula, radiofrequency or radioactive material. The first three methods involve the probable complication of haemorrhage deep in the brain substance, which at worst may be life-threatening or at least produce an area of brain destruction of unknown or unpredictable size. Radio-frequency lesions made by unipolar coagulation at a fixed temperature for a known time are reasonably predictable in size but may vary according to proximity to arteries and may be followed by secondary haemorrhage or other . 


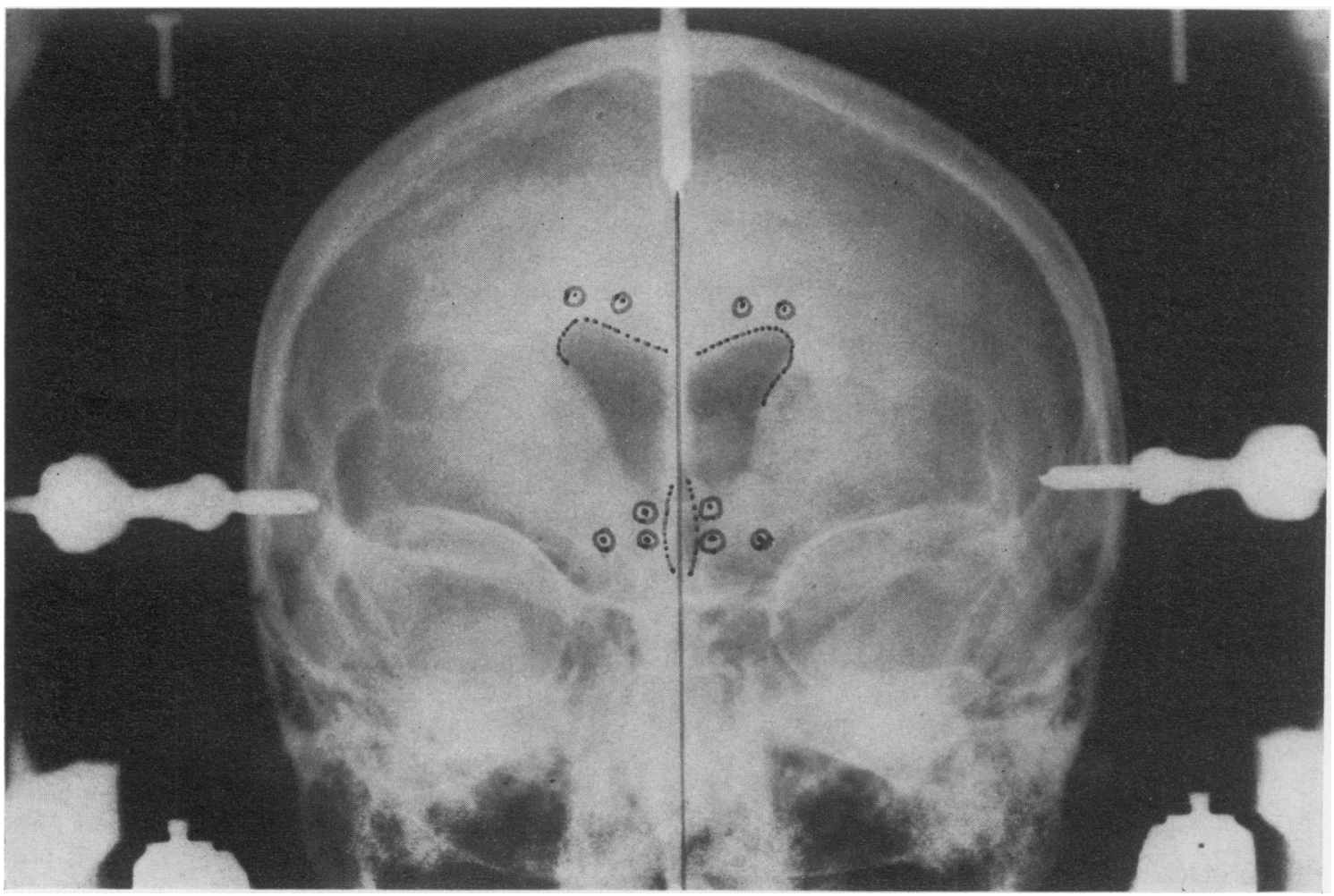

FIG. 3. Antero-posterior radiograph showing coincidence of the mid-line of the frame and the ventricular system. The target zones in the orbito-frontal region are seen as a triangle with the cingulum target zones plotted above the lateral ventricles.

vascular disturbance. It remains, however, a common technique used in many centres throughout the world. Radioactive ${ }^{90}$ Yttrium in the form of seeds is used by Knight (1965) for focal brain destruction in the orbital frontal region, but occasionally accurate placement and movement of the seeds may give rise to difficulties. We currently prefer the use of a cryogenic probe in most cases, though at times radiofrequency lesions are still used.

The probe is a double lumen device using nitrous oxide as the coolant. Its diameter is $3 \mathrm{~mm}$ and the un-insulated tip is $6 \mathrm{~mm}$ in length, incorporating a thermocouple for continuous monitoring of the tip temperature. The tip temperature is slowly reduced to $-70^{\circ} \mathrm{C}$ and this is maintained for $5 \mathrm{~min}$, after which slow temperature rise is allowed by regulating the gas pressure. Lesion size is to some extent dictated by the temperature fall, the time duration, the size of the probe and the rate of re-warming - the latter having the effect of increasing the lesion size if performed slowly. Using a standardized technique we have shown a consistent lesion size of 7-8 $\mathrm{mm}$ diameter in other surgically treated patients, where for the purpose of tumour removal or access it was felt necessary to remove a portion of brain and in whom it was possible to produce a cryogenic lesion prior to this removal. A vertical brain section is shown in Fig. 4 with a spherical dark lesion in the white matter deep to the cingulate gyrus some 4 months after surgery. Though some contraction in size has occurred the lesion still measures approximately $6 \mathrm{~mm}$ in diameter.

In referring to the target areas it was stated that seven lesion sites or target areas were plotted in each hemisphere, therefore our current regime in severe obsessional illness is to produce a total of fourteen lesions. A cautious approach in the early cases led us to make fewer lesions initially, and as was seen from Fig. 4 only one lesion is present in the cingulate region of the left hemisphere, whereas now there are four in each hemisphere. This gradual increase in the number of lesions required was dictated by a recurrence of symptoms in some of these earlier patients and the necessity for further operation with the production of additional lesions. This experience showed the benefits of the additional lesions which did not 


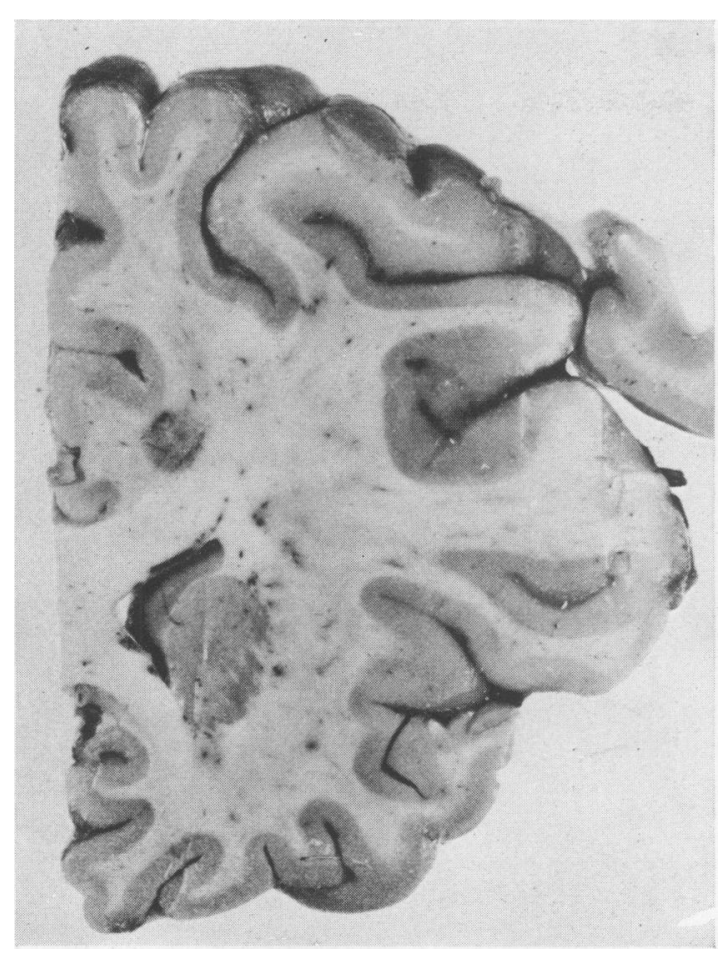

Fig. 4. A lesion $6 \mathrm{~mm}$ in diameter is shown in the cingulum bundle. By kind permission of Dr Marion Smith, MRC Unit at the National Hospital, Queen Square, London.

produce any detectable deficits in the patient. Confidence was, therefore, gradually acquired that this pattern of lesion was effective in symptom relief and in no way deleterious to the patient.

This technique, though time consuming, has the advantages of great accuracy of anatomical lesion placement with secondary target location depending upon the results of physiological responses to stimulation. The cryogenic probe can be entered into the brain substance without producing brain damage and its use results in a lesion of predictable size unaccompanied by any fear of secondary haemorrhage or reduction in the effective size of the lesion. In the earlier cases where further operation was deemed necessary it was possible, by employing the technique again, to place further lesions in accurate relationship to the preceding lesions and thus in a controlled way to increase slightly the extent of white fibre destruction. In the current series of cases there has been no mortality and only one case suffering a neurological complication, which was almost certainly due to traversing the head of the caudate nucleus. In this patient, who had previously had a rostral leucotomy, the orbito-frontal lesions had to be made more posteriorly than usual; this has led to a modification of burrhole placement, since which time no further difficulties have arisen.

\section{Post-operative management}

Rapid recovery of consciousness is the rule following surgery and thereafter routine neurological assessment is maintained at short intervals for $48 \mathrm{hr}$ and at increasing intervals thereafter. The routine follows the same pattern as that for any brain surgery. Bedrest is enforced for the first 2 or 3 days until the headache, due to the intracranial air, subsides. During the 5 to 7 days following surgery some confusion and disorientation is noted, incontinence is common and some delay in response is seen. An immediate lessening of tension is apparent but specific enquiry concerning obsessional symptoms is not usually made. The patient is thereafter ambulated and becomes independent. As the operation is prolonged, a course of antibiotics is given for 5 days and in view of the remote risk of epilepsy a course of anticonvulsants is prescribed for a period of $6^{\circ}$ months. One week after surgery the patient is transferred back to the care of the psychiatric department for the all important programme of rehabilitation.

\section{References}

KNIGHT, G.C. (1965) Stereotactic tractotomy in the surgical treatment of mental illness. Journal of Neurology, Neurosurgery and Psychiatry, 28, 304.

LEWIN, W. (1961) Observations on selective leucotomy. Journal of Neurology, Neurosurgery and Psychiatry, 24, 37.

Livingston, K.E. (1969) The frontal lobes revisited. Archives of Neurology, $20,90$.

NAUTA, W.J.H. (1962) Neural associations of the amygdaloid complex in the monkey. Brain, 85, 505 .

NAUTA, W.J.H. (1971) The problem of the frontal lobe: a re-interpretation. Journal of Psychiatric Research, 8, 167.

PAPEZ, J.W. (1937) A proposed mechanism of emotion. Archives of Neurology and Psychiatry, 38, 725. 